\title{
Clinical utility, safety, and efficacy of pregabalin in the treatment of fibromyalgia
}

\author{
This article was published in the following Dove Press journal: \\ Drug, Healthcare and Patient Safety \\ 17 February 2016 \\ Number of times this article has been viewed
}

\author{
Santosh Bhusal' \\ Sherilyn Diomampo' \\ Marina N Magrey ${ }^{2}$ \\ 'Division of Rheumatology, \\ Metrohealth Medical Center, ${ }^{2}$ Case \\ Western Reserve University School \\ of Medicine at Metrohealth Medical \\ Center, Cleveland OH, USA
}

Correspondence: Santosh Bhusal Metrohealth Medical Center, 2500 Metro health Drive, Cleveland, OH 44109, USA

Tel + I 2167785154

Fax + I 2167788376

Email sbhusal@metrohealth.org
Abstract: Fibromyalgia is a chronic debilitating medical syndrome with limited therapeutic options. Pregabalin, an anticonvulsant and $\alpha-2-\Delta$ subunit receptor ligand, is one of the anchor drugs approved by the US Food and Drug Administration for the treatment of fibromyalgia. The drug has shown clinically meaningful benefits across multiple symptom domains of fibromyalgia. Efficacy of pregabalin in fibromyalgia pain has been evaluated in at least five high-quality randomized trials, two long-term extension studies, a meta-analysis, a Cochrane database systematic review, and several post hoc analyses. These studies also hint towards a meaningful benefit on sleep, functioning, quality of life, and work productivity. Side effects of pregabalin, although common, are mild to moderate in intensity. They are noted early during therapy, improve or disappear with dose reduction, and are not usually life- or organ threatening. In most patients, tolerance develops to the most common side effects, dizziness, and somnolence, with time. With close clinical monitoring at initiation or dose titration, pregabalin can be effectively used in primary care setting. Pregabalin is cost saving with long-term use and its cost-effectiveness profile is comparable, if not better, to that of other drugs used in fibromyalgia. In the present era of limited therapeutic options, pregabalin undoubtedly retains its role as one of cardinal drugs used in the treatment of fibromyalgia. This review intends to discuss the clinical utility of pregabalin in the management of fibromyalgia with a focus on efficacy, safety, and cost-effectiveness.

Keywords: fibrositis, myofascial pain, diffuse pain syndrome, lyrica

\section{Introduction}

Fibromyalgia is a clinical syndrome characterized by chronic widespread pain (pain in all body quadrants - right and left side, above and below waist, as well as axial skeleton) associated with several other symptoms such as fatigue, waking unrefreshed, and cognitive complaints. Using the American College of Rheumatology (ACR) 1990 criteria, its prevalence is estimated to be $0.5 \%-4 \%{ }^{1}$ with $\sim 9: 1$ female-to-male ratio. It is the third most common rheumatologic condition after low back pain and osteoarthritis $^{2}$ and the most common reason for work-related disability leave in the developed world. ${ }^{3}$

The etiology and pathogenesis of fibromyalgia is uncertain. Genetic studies suggest an association with polymorphisms in serotonergic, dopaminergic, and catecholaminergic pathways involved in pain transmission and modulation. ${ }^{4}$ The disease is likely expressed in the presence of environmental trigger/triggers (eg, emotional trauma, acute illness, physical injury), ${ }^{5}$ which lead to expression of multiple genes that amplify pain perception in the pain processing pathway. Although a pain disorder 
by etymology, fibromyalgia is heterogeneous and may have several subgroups; these subgroups differ clinically and in response to drug therapy. ${ }^{6,7}$ Turk et $\mathrm{al}^{7}$ showed that patients with fibromyalgia could be classified into three subgroups based on multidimensional pain inventory self-reports: dysfunctional, interpersonally distressed, and adaptive copers. Similarly, Giesecke et $\mathrm{al}^{6}$ also purposed three subgroups based on predominant symptoms from one of the following domains: psychosocial (depression/anxiety), cognitive (catastrophizing/control over pain), and neurobiologic (tenderness). The heterogeneity of symptoms in pain syndromes including fibromyalgia could perhaps be better explained by the pain neuromatrix theory, ${ }^{8}$ which proposes the neurosignature of pain experience as one of the "action outputs" of brain from the so-called neuromatrix rather than a "passive response" to nociception (the traditional Cartesian view). Accordingly, the "action outputs" consist of pain perception with its sensory, affective, and cognitive domains in addition to voluntary as well involuntary action programs and stress regulation responses. In this context, an updated 2010 ACR preliminary diagnostic criteria for fibromyalgia which incorporates fatigue, waking unrefreshed, and cognitive symptoms in addition to pain and other somatic symptoms allow further cognizance of the multiple domains of this disease.

\section{Burden of fibromyalgia}

Fibromyalgia carries a global burden in patient's health - at individual, interpersonal, occupational, and socioeconomic levels. At an individual level, pervasive effects in all domains of health-related quality of life (HRQoL) measures are noted. In short form (SF) 36 questionnaires, patients with fibromyalgia have significant deficits in all eight components of physical and mental health compared to controls, ${ }^{9}$ whereas greater deficits in the scales measuring mental health (mental health, role limitations due to emotional health, social functioning, and vitality) over functional disability (physical functioning and role limitations due to physical function) $)^{9}$ are noted compared to rheumatoid arthritis patients. In interpersonal relationships, these patients receive less support from their partners compared to osteoarthritis patients and have higher caregiver burden at times of higher functional disability. ${ }^{10}$ Phenomenologically, fibromyalgia pain means living with chronic pain as well as pain during exacerbations with a simultaneous feeling that they are doubted because of the invisible nature of pain. ${ }^{11}$

At work, employees with fibromyalgia have a higher percentage of both work time missed (absenteeism) and onthe-job impairment (presenteeism) compared to patients with arthritis pain or back pain; they are two to three times more likely to miss work compared to normal controls. ${ }^{12,13}$ On average, they miss $15 \%$ of workdays (29.8 days in a year), which is higher than osteoarthritis patients ( 25.7 days; $P<0.0001){ }^{13}$ Furthermore, those with a high load of fibromyalgia symptoms are more likely to retire earlier than their asymptomatic twin counterparts (cumulative incidence: $25.7 \%$ vs $6.8 \%$, respectively). ${ }^{14}$ Receipt of social security disability in patients fulfilling the ACR 2010 criteria for fibromyalgia is comparable to patients with osteoarthritis and rheumatoid arthritis (42\% vs $42.4 \%$ vs $54.6 \%$, respectively). ${ }^{15}$

At a socioeconomic level, fibromyalgia is costly. Mean annual direct costs to the payers and patients amount to US\$7,973 (standard deviation [SD] US\$7,341) per subject in the USA, US\$2,234 (SD US\$2,261) in Germany, and US\$924 (SD US\$862) in France; the primary drivers of costs are prescription medications, physician office visits $(\sim 10$ / year), and out of pocket expenses. ${ }^{16}$ Indirect costs due to lost productivity, absenteeism, and disability are much higher and approximates about two-third to three-fourth of total costs. For example, mean annual indirect costs were US\$10,697 in the USA, US\$7,898 in Germany, and US\$9,819 in France..$^{13,16}$ Not surprisingly, the costs increase with increasing severity of disease. ${ }^{13}$

\section{Fibromyalgia and sleep}

Sleep difficulties are pervasive in fibromyalgia. In all, 88\% report sleep difficulty in one of the following domains: difficulty falling asleep, difficulty staying asleep, or waking too early; $63.05 \%$ report difficulty in two or more domains. ${ }^{17}$ Blocks of 2-3 hours of sleep followed by wakefulness in a "hyperarousal" mode (feeling very alert on awakening with difficulty going back to sleep for the next several hours) is the most common pattern. ${ }^{18}$ The experience of pain is in a bidirectional relationship with sleep disturbance - more sleep disturbance is associated with more pain and vice versa ${ }^{19}$ and is affirmed by an association of slow-wave sleep disruption with decreased pain threshold, increased fatigue, and increased discomfort in healthy volunteers. ${ }^{20} \mathrm{~A}$ path analysis model has demonstrated specific causal sequence involving sleep, pain, functional disability, and depression where baseline sleep predicted 1-year pain, baseline pain predicted 1 -year physical functioning, and baseline physical functioning predicted 1-year depression. ${ }^{21}$ Sleep disturbance, in addition, has an independent effect on HRQoL and disability, beyond any effect contributed by pain experience alone. ${ }^{22}$

Objective studies of sleep disturbance in fibromyalgia have yielded variable results. The original study of patients 
in "fibrositis" syndrome ${ }^{23}$ reported polysomnographic abnormalities that consist of delta waves periodically intruded by alpha waves (delta waves indicate restorative non-rapid eye movement (REM) sleep and alpha waves indicate drowsy wakefulness). Several other abnormalities have been reported since then which include disruption of sleep continuity from frequent arousals, reduced stage 2 sleep spindles, a shorter duration of stage 2 sleep, and high frequency of cyclical alternating pattern. ${ }^{24}$ Further studies are needed to discern the interrelationship between the abnormal sleep patterns and ascertain how they relate to pain, fatigue, and cognitive abnormalities in fibromyalgia.

\section{Current therapy of fibromyalgia}

Heterogeneity of symptoms in fibromyalgia deserves individualized treatment. Components of therapy include patient education, physical therapy, psychotherapy, and pharmacotherapy. Identifying potential subtypes based on predominant symptoms and customizing therapy may improve outcomes. ${ }^{25}$ Three medications are approved by the US Food and Drug Administration (FDA) for fibromyalgia - pregabalin, duloxetine, and milnacipran. A multimodal approach using one of the FDA-approved medications as an "anchor" drug, like methotrexate in rheumatoid arthritis, and subsequent tailoring of treatment with a "fatigue, insomnia, blues (depression), rigidity (stiffness), and Ow! (pain) (FIBRO)" model based on predominant symptom has been suggested. ${ }^{26}$ The ideal treatment strategy could be a combination of treatments, often in lower doses than used in clinical trials with medications such as modafenil (for fatigue), cyclobenazepril (for stiffness), zolpidem (for insomnia), and tramadol (for pain) added sequentially with a close monitoring for adherence and adverse events. ${ }^{27}$

\section{Pregabalin: mechanism of action and pharmacokinetics}

Pregabalin is a gamma-aminobutyric acid (GABA) analog. Although a structural analog, its mechanism of action does not directly relate to interaction with GABA receptor or significant alteration of GABA concentration in brain. ${ }^{28,29}$ Two different, and possibly interdependent, pathways of action are possible: presynaptic voltage-gated calcium channeldependent action and direct postsynaptic NMDA receptor activation. In the former, pregabalin binds with presynaptic $\alpha-2-\Delta$ subunit delta type 1 subunit of voltage-gated calcium channel and modifies the allosteric interaction between the calcium channel and presynaptic vesicle proteins (eg, syntaxin) reducing the ability of docked vesicles to fuse and release neurotransmitters. ${ }^{30}$ In regard to the latter, it was noted that postsynaptic NMDA receptor antagonists block pregabalin-mediated changes in vesicle release. ${ }^{30}$ Postsynaptic NMDA receptors may alter presynaptic transport of vesicles containing neurotransmitters using a nitric oxide pathway in which the newly synthesized nitric oxide (in response to postsynaptic NMDA receptor activation) diffuses to presynaptic membrane and alters vesicle trafficking. ${ }^{30}$ The final effect is a reduction of tonic release of several neurotransmitters, both excitatory and inhibitory, including GABA, which alters neuronal responsiveness leading to reduction of hyperexcitability in pain processing pathway. ${ }^{31}$

Pregabalin is rapidly absorbed after oral administration with a mean oral bioavailability of $90 \%{ }^{32}$ Time to reach maximum plasma concentration is $0.7-1.3$ hours and the elimination half-life is 4.6-6.6 hours. It is excreted unchanged in urine. ${ }^{32}$ A controlled release preparation of pregabalin (330 mg) once a day retains major pharmacokinetic profiles of $300 \mathrm{mg}$ pregabalin in terms of bioavailability and elimination half-life, ${ }^{33}$ which may potentially improve drug adherence.

\section{Clinical utility of pregabalin}

Clinical utility is a judgment about usefulness, benefits, and drawbacks of an intervention. A model for clinical utility ${ }^{34}$ has been postulated to consist of the following: appropriateness (is the intervention relevant? is it effective? is it safe?); accessibility (what are the costs and cost-effectiveness?); acceptability (is it acceptable to clinicians, patients, and public?); and practicability (does it do what we need it to do in actual everyday situations? are practitioners skilled and capable enough for its use or do they need further training?). These aspects will be discussed with a focus on efficacy, safety, and cost-effectiveness across various symptom domains (Table 1).

\section{Efficacy of pregabalin therapy: evidence from randomized trials}

The relevance of pregabalin therapy in fibromyalgia treatment cannot be overstated. It was the first medicine approved by the FDA for fibromyalgia treatment in 2007. Since then, only two other medications, duloxetine and milnacipran, have been approved. The usefulness of pregabalin as an "anchor drug", discussed earlier, is relevant not only for its benefit in pain, but also for its benefits in sleep and HRQoL.

\section{Efficacy in treatment of pain}

Several randomized trials and two meta-analyses have demonstrated the efficacy of pregabalin in treatment of pain in 


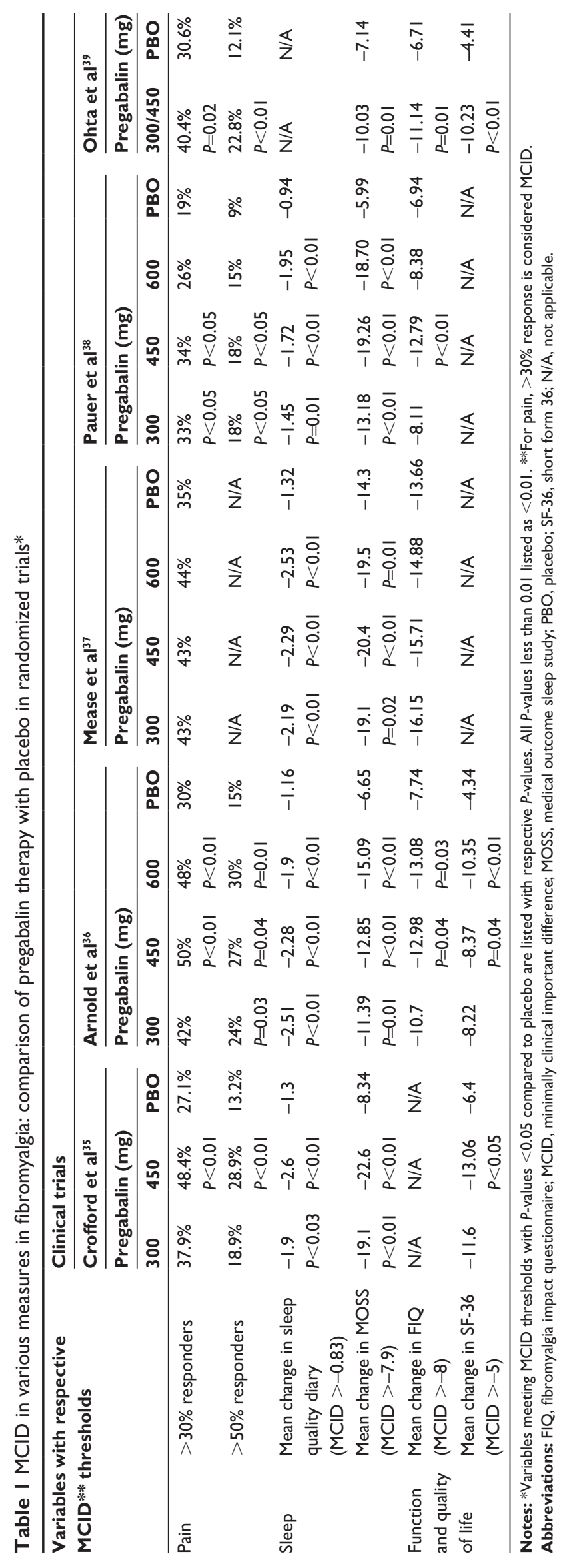


fibromyalgia. Most trials $\mathrm{s}^{35-37}$ involved a sample of: $>90 \%$ white females who meet the ACR 1990 criteria for fibromyalgia; average age of 49-50 years; and median pain score of 6.7-7.1/10. In this context, a multicenter double-blind trial $^{35}$ with 529 participants randomized to take 150, 300, and $450 \mathrm{mg}$ of pregabalin or placebo for 8 weeks demonstrated that patients taking pregabalin $450 \mathrm{mg}$ had significant improvement in pain as evidenced by: 1) reduction of endpoint mean pain scores at all weeks from 1 to 7 (baseline mean pain score 6.9 for placebo and 7 for pregabalin $450 \mathrm{mg}$ / day; least square mean pain score at week $8: 5.88$ for placebo and 4.94 for pregabalin $450 \mathrm{mg}$ /day; mean change: -1.02 for placebo and -1.96 for pregabalin $450 \mathrm{mg} /$ day $[P=0.0009])$ and 2) reports of more than $50 \%$ improvement of pain from baseline (28.9\% pregabalin vs $13.2 \%$ placebo; $P=0.003$ ). However, there was no significant difference in the endpoint mean pain scores and the proportion of patients achieving $>30 \%$ reduction of pain in patients taking 300 and $150 \mathrm{mg}$ of pregabalin compared to placebo. Another randomized, double-blind control trial ${ }^{36}$ involving 745 patients which used a higher dose of pregabalin $(300,450$, and $600 \mathrm{mg}$ ) for a period of 14 weeks revealed a statistically significant reduction of endpoint mean pain scores in all treatment groups compared to placebo (baseline pain scores: 6.6, 6.7, 6.6, and 6.7 for placebo and pregabalin 300, 450, and $600 \mathrm{mg} /$ day, respectively; least square mean pain scores at week 14: 5.64, 4.93, 4.66, and 4.64 for placebo and pregabalin 300, 450, and $600 \mathrm{mg} /$ day, respectively; mean change -1.04 for placebo, -1.75 for pregabalin $300 \mathrm{mg} /$ day [ $P=0.009],-2.03$ for pregabalin $450 \mathrm{mg} /$ day $[P<0.001]$, and -2.05 for pregabalin $600 \mathrm{mg} /$ day $[P<0.001])$. The proportion of patients achieving $>50 \%$ reduction of pain from baseline was also significantly higher in all treatment groups (15\% for placebo, $24 \%$ for $300 \mathrm{mg} /$ day, $27 \%$ for $450 \mathrm{mg} /$ day, and $30 \%$ in $600 \mathrm{mg} /$ day $[P=0.0372, P=0.038$, and $P=0.010$, respectively $]$ ) as was the proportion of patients achieving $>30 \%$ improvement $(30 \%$ for placebo, $42 \%$ for $300 \mathrm{mg} /$ day, $50 \%$ for $450 \mathrm{mg} /$ day, and $48 \%$ for $600 \mathrm{mg} /$ day $[P=0.0172, P=0.002$, and $P=0.006$, respectively]). A very similar study ${ }^{37}$ of 748 patients randomized to take similar dosages of pregabalin (300, 450, and $600 \mathrm{mg}$ ) or placebo for 13 weeks revealed statistically significant improvement in endpoint mean pain scores for all the dosages compared with placebo (baseline mean pain scores: 7.2, 7.1, 7.1, and 7 for placebo and pregabalin 300, 450 , and $600 \mathrm{mg} /$ day, respectively; mean pain scores at week 13: 5.7, 5.26, 5.23, and 5.04 for placebo and pregabalin 300, 450 , and $600 \mathrm{mg} /$ day, respectively; mean change -1.4 for placebo, -1.84 for pregabalin $300 \mathrm{mg} /$ day $[P=0.0449],-1.87$ for pregabalin $450 \mathrm{mg} /$ day $[P=0.0449]$, and -2.06 for pregabalin $600 \mathrm{mg} /$ day $[P=0.007])$. In this study, however, statistical significance was not reached between the pregabalin-treated patients and placebo in terms of the proportion of patients achieving $>30 \%$ reduction in pain. Nevertheless, there was a trend toward higher proportion of patients achieving $>30 \%$ reduction in pain with pregabalin therapy $(43 \%, 43 \%$, and $44 \%$ among patients receiving 300, 450, and $600 \mathrm{mg} /$ day of pregabalin, respectively) compared to placebo (35\%).

An international, randomized, double-blinded trial ${ }^{38}$ evaluated the efficacy of pregabalin in 747 patients, randomly assigned to receive 300,450 , and $600 \mathrm{mg}$ /day of pregabalin or placebo (a total of 73 centers across Europe, Canada, Asia, and Australia). The patient population was more diverse but still representative of general population with fibromyalgia (51\% from European countries, 49\% non-European; meeting the ACR 1990 criteria, 91\% females, 76\% white, mean age 48.5 years, mean duration of fibromyalgia 8.2 years, and baseline mean pain score of 6.65/10). Statistically significant reduction on endpoint mean pain scores were noted only in the patients taking pregabalin $450 \mathrm{mg} /$ day (baseline mean pain scores: $6.68,6.76,6.59$, and 6.65 for placebo and pregabalin 300,450 , and $600 \mathrm{mg} /$ day, respectively; mean pain scores at week 14: 5.92, 5.59, 5.36, and 5.69 for placebo and pregabalin 300,450 , and $600 \mathrm{mg} /$ day, respectively; mean change: -0.73 for placebo and -1.29 for pregabalin $450 \mathrm{mg} /$ day $[P=0.0132]$ ) when last observation carried forward method was used. However, with duration adjusted average change sensitivity analysis, all three dosages showed significant reduction of the endpoint mean pain scores (mean difference with placebo: -0.47 for pregabalin $300 \mathrm{mg} /$ day [ $P=0.0024]$; -0.61 for pregabalin $450 \mathrm{mg} /$ day $[P<0.0001]$; and -0.47 for pregabalin $600 \mathrm{mg} /$ day $[P=0.0023])$. Patients achieving $>30 \%$ reduction with 300,450 , and $600 \mathrm{mg} /$ day of pregabalin were $33 \%, 34 \%$, and $26 \%$, respectively, as compared to $19 \%$ for placebo; those achieving $>50 \%$ reduction of pain with 300,450 , and $600 \mathrm{mg}$ of pregabalin were $18 \%, 18 \%$, and $15 \%$, respectively, as compared to $9 \%$ for placebo. With pregabalin 300 and $450 \mathrm{mg} /$ day and placebo, the difference was statistically significant $(P<0.05)$ for both $>30 \%$ and $>50 \%$ responders but with $600 \mathrm{mg} /$ day, it was not. Similarly, a randomized double-blind trial involving 498 Japanese patients ${ }^{39}$ (meeting the ACR criteria, $89 \%$ females, mean age 47 years, mean disease duration 6.5 years, mean pain scores 6.5) using 300 or $450 \mathrm{mg}$ /day of pregabalin revealed a statistically significant improvement of endpoint mean pain scores at 15 weeks of treatment (baseline pain score 6.4 and 6.5 for placebo and pregabalin groups, respectively; mean pain scores at week 15: 5.45 and 5.01 for 
placebo and pregabalin, respectively; mean change: -1.03 for placebo and -1.48 for pregabalin, $P=0.0046)$. Similar to most prior studies, patients achieving $>50 \%$ and $>30 \%$ improvement in pain were significantly higher in pregabalin group compared to placebo (50\% responders: $22.8 \%$ and $12.1 \%$ in pregabalin and placebo, respectively, $P=0.0017$; $30 \%$ responders: $40.4 \%$ and $30.6 \%$ in pregabalin and placebo, respectively, $P=0.0230$ ). The latter study also had a 53 -week open-label extension phase to address the long-term efficacy and safety, which will be discussed later.

The fibromyalgia relapse evaluation and efficacy for durability of meaningful relief (FREEDOM) trial ${ }^{40}$ enriched for responders with placebo-controlled discontinuation evaluated the durability of response in pregabalin-treated patients. An initial 6-week open-label treatment $(n=1,051)$ phase was carried out for optimization of dosage $(300,450$, and $600 \mathrm{mg} /$ day) and identification of responders (which was defined by $>50 \%$ reduction in pain visual analog scale [VAS] from baseline and a self-rating of overall improvement on the patient global impression of change scale of "much improved" or "very much improved"). Responders $(n=566$ of 1,051$)$ then entered a double-blind phase where they were randomized to continue pregabalin or receive placebo for 26 weeks. The primary endpoint was time to loss of therapeutic response measured in days, and defined as either $<30 \%$ reduction in pain VAS score relative to openlabel baseline value at two consecutive visits or worsening of fibromyalgia necessitating alternative treatment. Among 566 randomized patients, 162 completed the trial $(19 \%$ for placebo; $48 \%, 33 \%$ and $37 \%$ for 300,450 , and $600 \mathrm{mg} /$ day, respectively). The time to loss of therapeutic response was significantly longer for all pregabalin-treated patients as compared to placebo (half of placebo-treated group had loss of therapeutic response by day 19 whereas more than half of all pregabalin-treated patients still had not lost therapeutic response at the end of the trial). At the end of the trial, 61\% of placebo-treated patients had lost therapeutic response as compared to $32 \%$ in pregabalin-treated patients $(P<0.001)$. An open-label extension study from Japan ${ }^{41}$ involving 106 patients also revealed similar findings in regard to the durability of response. In this study, the mean pain VAS scores at week $15(4.8 / 10)$ and week 53 (4.9/10) were similar; only $6.6 \%$ of the patients had discontinued pregabalin due to lack of therapeutic response. Hence, most patients who initially respond to pregabalin (which may begin as early as 1 week of therapy, when responders start to separate from placebo in randomized trials ${ }^{38-40}$ ) will continue to do so and loss of therapeutic response over time is unlikely.
In reference to the benefits in fibromyalgia pain, it has to be noted that a 2-point numeric reduction in the VAS scale $(0-10)$ or $>30 \%$ reduction in pain from baseline represents a clinically meaningful difference ${ }^{42}$ (Table 1 ). In regard to the former, the endpoint mean pain scores after treatment with pregabalin closely approached this threshold in most of the aforementioned trials ${ }^{35-37,39}$ (range: -1.29 to -2.06 ), especially with dosages higher than $450 \mathrm{mg}$ /day. In regard to the latter, a meta-analysis of clinical trials of pregabalin in fibromyalgia ${ }^{43}$ and a Cochrane database systematic review on the use of pregabalin in acute and chronic pain, ${ }^{44}$ including fibromyalgia, revealed that on an average approximately $30 \%-50 \%$ of patients treated with pregabalin were able to achieve $>30 \%$ reduction of pain from baseline and $20 \%-30 \%$ patients achieved $>50 \%$ reduction of pain. The number needed to treat ranged from 9 to 14 . Furthermore, in the Cochrane analysis, ${ }^{44}$ the following were discerned: 1) pregabalin $150 \mathrm{mg} /$ day was not different from placebo in efficacy; 2) greater response was noted with higher dosages up to $450 \mathrm{mg} /$ day, with lower (better) number needed to treat values; 3) pregabalin $600 \mathrm{mg} /$ day did not produce better results than $450 \mathrm{mg}$; and 4 ) the relative benefit for $>30 \%$ or $>50 \%$ pain reduction with pregabalin $450 \mathrm{mg}$ /day was 1.5 as compared to 1.1 for placebo. Although only about a third of total patients were able to achieve the clinically meaningful benefit in terms of pain response, it does represent an important difference for those who achieve this.

\section{Efficacy in treatment of sleep disturbance}

It is intriguing that pregabalin enhances slow-wave sleep. A double-blind study involving 12 healthy volunteers compared the effects on sleep of pregabalin $450 \mathrm{mg}$ /day to alprazolam and placebo. ${ }^{45}$ Pregabalin-treated patients had a higher proportion of delta wave sleep (stage 3 and stage 4 ) compared to both placebo and alprazolam $(\sim 36 \%, 18 \%$, and $25 \%$ of total sleep time on night 3 , for pregabalin, alprazolam, and placebo, respectively $(P<0.001))$; this effect was evident in all thirds of the night. This was in contrast to alprazolam which decreased slow-wave sleep in the second and final thirds of night and increased the proportion of stage 2 sleep. Pregabalin therapy was also associated with fewer awakenings of longer than 1-minute duration. Another randomized, placebo-controlled two-way crossover polysomnography study ${ }^{23}$ involving 119 patients with fibromyalgia with a history of disturbed sleep (subjective total sleep time $<6$ hours/night and subjective wake after sleep onset [WASO] $>60$ minutes for $>3$ nights/week) with objective polysomnographic abnormalities of WASO $>45$ minutes and total sleep time of 
3-6.5 hours for 2 consecutive nights revealed a reduction of WASO by 19 minutes after 4 weeks of treatment (WASO for pregabalin 51.5 vs 70.7 minutes for placebo $[P<0.0001])$ and increase in total sleep time by $>25$ minutes (total sleep time 396 and 370 minutes for pregabalin and placebo, respectively $[P<0.0001])$. Hence, pregabalin may improve the quality as well as the quantity of sleep.

Benefits of pregabalin therapy on sleep are well translated in clinical trials. Clinically meaningful improvement in sleep quality corresponds to a change of $10 \mathrm{~mm}$ on sleep quality variable on Leeds Sleep Evaluation Questionnaire VAS, ${ }^{46}$ 0.83 on sleep quality diary, ${ }^{47}$ and 7.9 units on medical outcome sleep study ${ }^{48}$ (Table 1). In an analysis of two randomized trials in fibromyalgia, ${ }^{47}$ patients taking pregabalin had statistically significant improvements in both sleep quality diary and medical outcome sleep study, the difference with placebo exceeding the thresholds for clinical meaningfulness (0.86-1.35 for sleep quality diary and $8.91-14.93$ for medical outcome sleep study); these results were more consistent at doses $450 \mathrm{mg} /$ day or higher (Table 1). Using a mediation analysis model, the study ${ }^{47}$ concluded that $43 \%-80 \%$ of improvement of sleep was due to medication itself and $20 \%-57 \%$ of the improvement was mediated via improvement in pain. Beneficial effects on sleep can be seen as early as day 1 and median time to sustained clinical improvement is 11 days. ${ }^{49}$ Durability of benefit was also evaluated by a post hoc analysis of FREEDOM trial, where median time to loss of therapeutic response was 41 days for placebo, whereas $>50 \%$ of pregabalin-treated patients continued to maintain therapeutic response at week $26 .{ }^{50}$ Thus, meaningful benefit in sleep is lasting and there is little evidence of significant tolerance.

\section{Efficacy in functioning and quality of life}

The fibromyalgia impact questionnaire (FIQ) and SF-36 health survey are the most commonly used measures for functioning and HRQoL. Significant improvements in FIQ were noted with pregabalin at the doses of 450 and $600 \mathrm{mg} /$ day compared to placebo in a randomized trial ${ }^{36}$ (baseline FIQ scores: 58.7, 61.1, 59.6, and 59.5 for placebo and pregabalin 300,450 , and $650 \mathrm{mg} /$ day, respectively; FIQ scores at week 14: 51.99, 49.03, 46.75, and 46.65 for placebo and pregabalin 300, 450, and $600 \mathrm{mg} /$ day, respectively; mean change from baseline: -7.74 for placebo, -12.98 for pregabalin $450 \mathrm{mg} /$ day $[P=0.0041]$ and -13.08 for pregabalin $600 \mathrm{mg} /$ day $[P=0.0034]$ ); the difference did not reach statistical significance in patients taking the dose of $300 \mathrm{mg}$ /day (FIQ score at baseline: 61.1 and at week 13:
49.03; mean change: -10.70 vs -7.74 for placebo $[P=0.1]$ ). Another randomized tria ${ }^{38}$ showed significant improvement in FIQ with the pregabalin dose of $450 \mathrm{mg}$ /day only (baseline FIQ score 62.58 and 60.59 for placebo and pregabalin $450 \mathrm{mg}$ /day, respectively; FIQ scores at week 14: 54.14 for placebo 48.29 for pregabalin $450 \mathrm{mg} /$ day; mean change: -6.74 for placebo and -12.79 for pregabalin $450 \mathrm{mg} /$ day $[P=0.0012]$ ) but not with 300 and $600 \mathrm{mg} /$ day (baseline FIQ scores: 60.69 and 60.4, respectively; FIQ scores at week 14: 52.97 and 52.7, respectively; mean change -8.11 and -8.38 $[P=0.5$ and 0.4 , respectively]). A third randomized trial with a similar sample size,${ }^{37}$ however, failed to show a statistically significant response in FIQ although there was an overall trend toward improvement (baseline FIQ scores: 64.3 for all groups; FIQ at week 13: 50.66, 48.18, 48.62, and 49.45 for placebo and pregabalin 300, 450, and $600 \mathrm{mg} /$ day, respectively; mean change: $-13.66,-16.15,-15.71$, and -14.88 for placebo, pregabalin 300,450 , and $600 \mathrm{mg} /$ day, respectively: all $P$-values $>0.2$ ). A study from Japan revealed the most remarkable improvement in the following domains of FIQ: waking up well rested, feeling good, fatigue, and pain. ${ }^{39}$ Considering a change of 8 points in FIQ to be a minimal clinically important difference, ${ }^{50}$ almost all the dosages of pregabalin seem to exceed this outcome while the effect of placebo was closely approaching ${ }^{36,38}$ and, indeed, exceeding in one study ${ }^{37}$ (Table 1). Although only a modest improvement from placebo, this difference does have a clinical meaningfulness as discussed.

SF-36 health survey results are diverse. Significant improvements in several domains of SF-36 are demonstrated but are not consistent across different studies. Improvement was noted on the following domains: social functioning, ${ }^{35,36}$ bodily pain, ${ }^{34}$ vitality, $, 35,36,39$ general health, ${ }^{35}$ physical functioning, ${ }^{39}$ and mental health ${ }^{36,39}$ with all studies showing improvement in SF-36 vitality scale. Considering a change of 5 units on SF-36 vitality scale equated with a minimally important clinical difference, ${ }^{50}$ all studies ${ }^{35,36,39}$ achieved this consistently (Table 1 ). The FREEDOM tria ${ }^{40}$ demonstrated the persistence of beneficial effects for up to 6 months in the majority of patients who initially responded to therapy. Hence, meaningful benefit in quality of life, at least on the vitality scale, may be achieved by pregabalin therapy. In addition, there are beneficial effects in ergonomics. In a meta-analysis of four randomized trials,${ }^{51}$ patients who had VAS pain score $<30 \mathrm{~mm}$ at the end of trial and $>50 \%$ improvement of pain from baseline (double responders) had $67 \%$ reduction in work interference (both presenteeism and absenteeism), those with endpoint VAS pain score $<30 \mathrm{~mm}$ 
only had $55 \%$ reduction, and those with $>50 \%$ improvement only had $27 \%$ reduction (as compared to $12 \%$ with reduction in nonresponders; all $P$-values $<0.001)$. Approximately 1.4 days of work per week was gained in double responders, 1.2 days/week in patients achieving VAS pain score of $30 \mathrm{~mm}$ or less, and 1 day/week in patients with $>50 \%$ improvement in pain. As a result of this, improved productivity and perhaps a reduction of indirect costs of fibromyalgia could ensue.

\section{Safety}

Side effects of pregabalin treatment are common but do not interfere with functions of daily life. The rate of discontinuation is low. Treatment emergent adverse events are noted in $\sim 80 \%-90 \%$ of patients in pregabalin-treated group and approximately $70 \%-75 \%$ in placebo group in randomized trials. ${ }^{35,36,39}$ Among the side effects attributed to pregabalin therapy, the most common are dizziness and somnolence with relative frequency of approximately $40 \%-50 \%$ and $30 \%-45 \%$, respectively. ${ }^{35-39}$ The median time to onset for each of these is 1-2 days. ${ }^{35,37}$ In patients, who continued pregabalin, the median duration of dizziness was 4-44 days (longer duration for those taking higher dose, ie, dose-related) and the median duration of somnolence was 18-88 days (not dose-related). ${ }^{35,37,43}$ Overall, 3\%-9\% of patients discontinue pregabalin due to dizziness and a further 3\%-6\% withdraw due to somnolence. ${ }^{35-38}$ In long-term extension studies, the prevalence of dizziness and somnolence decreases by about half, where $17 \%$ continue to complain of dizziness and approximately $8 \%$ continue to complain of somnolence. ${ }^{52}$ This is thought to be related to the development of tolerance to these side effects. ${ }^{52}$

Weight gain and peripheral edema are less common than dizziness and somnolence but can lead to discontinuation of therapy. Approximately $14 \%$ of pregabalin-treated patients complain of weight gain at 3 months of therapy and the incidence of clinically significantly weight gain $(>7 \%$ weight gain) is approximately $10 \%$. The severity of weight gain is usually deemed as mild to moderate and approximately $2 \%$ of patients discontinue treatment. ${ }^{36-38}$ However, the incidence of weight gain has been reported to be as high as $18 \%-26 \%$ in a year in some long term studies, ${ }^{39,52}$ which may further lead to discontinuation of therapy. Peripheral edema, on the other hand, is experienced by approximately $8 \%-11 \%$ of patients at 3 months of therapy. ${ }^{35-38}$ Discontinuations due to peripheral edema is rare $(<0.5 \%)$ and there is no incremental incidence at 1 year compared to 3 months. ${ }^{35-38,41,52}$ No changes in cardiovascular and renal function were noted in patients with peripheral edema. ${ }^{35} \mathrm{~A}$ handful of case reports of worsening of preexisting heart failure ${ }^{53-55}$ due to fluid retention are available in literature. This has led to a proposal of a systematic analysis for evaluation of the strength of association between heart failure and pregabalin therapy, ${ }^{56}$ the results of which are actively awaited. In the interim, the New York Heart Association has issued a warning about being careful when prescribing pregabalin in patients with New York Heart Association class 3 and 4 heart failure. ${ }^{57}$

Central adverse events associated with pregabalin therapy with incidence higher than $5 \%$ include blurring of vision, disturbance of attention, balance issues, vertigo, incoordination, thinking abnormally, confusion, euphoria, and impaired attention. These rarely lead to discontinuation of therapy and are also deemed mild to moderate in severity. ${ }^{35-38}$ Noncentral adverse effects of incidence higher than placebo include dry mouth, increased appetite, and constipation, which are also usually mild. ${ }^{35-38}$ Most of these adverse effects were noted with pregabalin when used as an anticonvulsant before its use in fibromyalgia. Reassuringly, no new patterns of adverse events were noted with its use in fibromyalgia patients.

The serious adverse events leading to discontinuation of pregabalin therapy are mostly related to dizziness and somnolence. ${ }^{35-38}$ Other extremely rare events, with prevalence in the range of $0.1 \%$, described in patients taking pregabalin which led to discontinuation of therapy, include myoclonus, chest pain, crush injury, motor vehicle accidents, abnormal liver function tests, and suicidal ideation. ${ }^{36-39,52}$ Because of the rarity of these events with only one to two events per randomized trial, the causal association is difficult to establish. Moreover, corresponding investigators of the trials have concluded that almost all these events were unrelated to pregabalin therapy.

No significant differences were noted between placebo and pregabalin-treated patients in blood pressure, pulse rates, serum chemistry, liver function tests, electrocardiogram (EKG), or urinalysis. ${ }^{35-38} \mathrm{~A}$ mild increase in creatinine kinase was noted in Japanese patients $(2.8 \%$ with pregabalin vs $0.4 \%$ with placebo) but this did not lead to discontinuation of therapy. ${ }^{39}$ It has to be noted that all trials were conducted in patients with a Glomerular function rate (GFR) of more than $60 \mathrm{~mL} / \mathrm{minute} / 1.73 \mathrm{~m}^{2}$ except in one trial where the dose was halved in patients with a GFR of $30-60 \mathrm{~mL} /$ minute $/ 1.73$ $\mathrm{m}^{2.41}$ no significant increase in adverse events were noted in the latter. To date, the safety of pregabalin in fibromyalgia patients with a GFR less than $30 \mathrm{~mL} /$ minute $/ 1.73 \mathrm{~m}^{2}$ has not been evaluated.

In summary, pregabalin-treated patients have moderate to high incidence of side effects; the side effects are usually mild and serious adverse events are extremely 
rare. The rate of discontinuation of therapy due to adverse event is not uncommon and is more likely with a higher dose with relative risk of 1.6 for $300 \mathrm{mg}$ /day and 2.5 for $600 \mathrm{mg} /$ day. ${ }^{44}$ Many patients develop tolerance to the side effects with continuation of therapy. There is no need for regular monitoring of blood chemistry or hematology and clinical monitoring can usually be accomplished in the primary care setting.

\section{Accessibility: cost-effectiveness}

Pharmacoeconomic studies reveal that pregabalin is costeffective. A Marcov ${ }^{58}$ model for the assessment of costeffectiveness of pregabalin in the USA noted that at 12 weeks, the total and indirect costs were lower in patients taking pregabalin 300 and $450 \mathrm{mg}$ /day compared to placebo. The direct costs (outpatient visits and medications) were, however, slightly higher. In this instance, pregabalin $450 \mathrm{mg}$ /day was cost saving overall, but pregabalin $300 \mathrm{mg}$ /day was not. Nevertheless, at 1 year, both the dosages of pregabalin were cost saving; the total, direct and indirect, costs were all lower than that of placebo. ${ }^{58}$ Pregablin was also cost saving as compared to duloxetine, gabapentin, and milnacipran. ${ }^{58} \mathrm{~A}$ study from $\mathrm{UK}^{59}$ revealed similar findings where pregabalin was found to be cost-effective at a threshold of $£ 30,000$ (approximately US\$48,000) per QALY gained (in general, an intervention is considered cost-effective at a threshold of US\$50,000 or less per QALY gained $\left.{ }^{60}\right)$. In both studies, ${ }^{58,59}$ the incremental cost-effectiveness ratios with pregabalin therapy was highest in patients with severe fibromyalgia and with $450 \mathrm{mg}$ /day dosage. Studies from Iran $^{61}$ and Mexico ${ }^{62}$ revealed consistent effects on cost saving, with most benefit with pregabalin 450 and $600 \mathrm{mg} /$ day. ${ }^{61}$ Comparative analyses of pregabalin and other drugs in fibromyalgia, especially duloxetine, have not always revealed consistent results with some studies pointing toward higher health care resource use with pregabalin, probably driven by more inpatient and outpatient visits and medication use, while others revealed nonsignificant difference between the two. ${ }^{63}$ Almost all cost-effectiveness analyses are dominated by amitriptyline because of its lower costs but the comparison between amitriptyline and pregabalin relies on older studies which may not meet current quality criteria ${ }^{59}$ and the degree of overall clinical improvement (reduction of VAS score by $>50 \%$ and decrease in FIQ score by $30 \%$ ) is lower with amityrptyline. ${ }^{62}$ It may thus be stated that pregabalin therapy results in better outcomes at a higher cost when compared to amitriptyline. To conclude, long-term pregabalin therapy is certainly cost-effective in fibromyalgia especially at doses $450 \mathrm{mg} /$ day or higher and at least cost neutral, if not cost saving, with $300 \mathrm{mg} /$ day dose and is comparable to other drugs used in fibromyalgia.

\section{Conclusion}

Fibromyalgia, a chronic disease with poorly understood pathophysiology, carries a substantial burden in patients as well as the society. Pregabalin therapy in patients with fibromyalgia is modestly effective in terms of response, but a good number of patients are able to achieve meaningful benefit in terms of pain control, improvement of sleep, functioning, and quality of life. Side effects from pregabalin therapy, although common, are usually mild, well tolerated in long-term, and can be monitored in primary care setting. Pregabalin is cost saving to the society, especially if used at dosages $450 \mathrm{mg} /$ day or higher. Until the availability of better drugs in future, pregabalin is well suited to serve as one of the "anchor drugs" in fibromyalgia.

\section{Disclosure}

The authors report no conflicts of interest in this work.

\section{References}

1. Wolfe F, Ross K, Anderson J, Russell IJ. Aspects of fibromyalgia in the general population: sex, pain threshold, and fibromyalgia symptoms. J Rheumatol. 1995;22(1):151-156.

2. Lawrence RC, Felson DT, Helmick CG, et al. Estimates of the prevalence of arthritis and other rheumatic conditions in the United States. Part II. Arthritis Rheum. 2008;58(1):26-35.

3. SkaerTL. Fibromyalgia: disease synopsis, medication cost effectiveness and economic burden. Pharmacoeconomics. 2014;32(5):457-466.

4. Buskila D, Sarzi-Puttini P. Biology and therapy of fibromyalgia. Genetic aspects of fibromyalgia syndrome. Arthritis Res Ther. 2006; 8(5):218.

5. Bennett RM, Jones J, Turk DC, Russell IJ, Matallana L. An internet survey of 2,596 people with fibromyalgia. BMC Musculoskelet Disord. 2007;8:27.

6. Giesecke T, Williams DA, Harris RE, et al. Subgrouping of fibromyalgia patients on the basis of pressure-pain thresholds and psychological factors. Arthritis Rheum. 2003;48(10):2916-2922.

7. Turk DC, Okifuji A, Sinclair JD, Starz TW. Pain, disability, and physical functioning in subgroups of patients with fibromyalgia. J Rheumatol. 1996;23(7):1255-1262.

8. Melzack R. Evolution of the neuromatrix theory of pain. The Prithvi Raj Lecture: presented at the third World Congress of World Institute of Pain, Barcelona 2004. Pain Pract. 2005;5(2):85-94.

9. Salaffi F, Sarzi-Puttini P, Girolimetti R, Atzeni F, Gasparini S, Grassi W. Health-related quality of life in fibromyalgia patients: a comparison with rheumatoid arthritis patients and the general population using the SF-36 health survey. Clin Exp Rheumatol. 2009;27(5 Suppl 56):S67-S74.

10. Reich JW, Olmsted ME, van Puymbroeck CM. Illness uncertainty, partner caregiver burden and support, and relationship satisfaction in fibromyalgia and osteoarthritis patients. Arthritis Rheum. 2006;55(1):86-93.

11. Juuso P, Skär L, Olsson M, Söderberg S. Living with a double burden: meanings of pain for women with fibromyalgia. Int J Qual Stud Health Well-being. 2011;6(3):7184.

12. McDonald M, DiBonaventura Md, Ullman S. Musculoskeletal pain in the workforce: the effects of back, arthritis, and fibromyalgia pain on quality of life and work productivity. J Occup Environ Med. 2011;53(7):765-770. 
13. White LA, Birnbaum HG, Kaltenboeck A, Tang J, Mallett D, Robinson RL. Employees with fibromyalgia: medical comorbidity, healthcare costs, and work loss. J Occup Environ Med. 2008;50(1):13-24.

14. Markkula R, Kalso E, Huunan-Seppälä A, et al. The burden of symptoms predicts early retirement: a twin cohort study on fibromyalgia-associated symptoms. Eur J Pain. 2011;15(7):741-747.

15. Wolfe F, Walitt BT, Katz RS, Häuser W. Social security work disability and its predictors in patients with fibromyalgia. Arthritis Care Res (Hoboken). 2014;66(9):1354-1363.

16. Knight T, Schaefer C, Chandran A, Zlateva G, Winkelmann A, Perrot S. Health-resource use and costs associated with fibromyalgia in France, Germany, and the United States. Clinicoecon Outcomes Res. 2013;5:171-180.

17. Bigatti SM, Hernandez AM, Cronan TA, Rand KL. Sleep disturbances in fibromyalgia syndrome: relationship to pain and depression. Arthritis Rheum. 2008; 59(7):961-967.

18. Winkelmann A, Perrot S, Schaefer C, et al. Impact of fibromyalgia severity on health economic costs: results from a European cross-sectional study. Appl Health Econ Health Policy. 2011; 9(2):125-136.

19. Theadom A, Cropley M. 'This constant being woken up is the worst thing' - experiences of sleep in fibromyalgia syndrome. Disabil Rehabil. 2010;32(23):1939-1947.

20. Affleck G, Urrows S, Tennen H, Higgins P, Abeles M. Sequential daily relations of sleep, pain intensity, and attention to pain among women with fibromyalgia. Pain. 1996;68(2-3):363-368.

21. Lentz MJ, Landis CA, Rothermel J, Shaver JL. Effects of selective slow wave sleep disruption on musculoskeletal pain and fatigue in middle aged women. J Rheumatol. 1999;26(7):1586-1592.

22. Wagner JS, DiBonaventura MD, Chandran AB, Cappelleri JC. The association of sleep difficulties with health-related quality of life among patients with fibromyalgia. BMC Musculoskelet Disord. 2012;13:199.

23. Moldofsky H, Scarisbrick P, England R, Smythe H. Musculoskeletal symptoms and non-REM sleep disturbance in patients with "fibrositis syndrome" and healthy subjects. Psychosom Med. 1975;37(4):341-351.

24. Roth T, Lankford DA, Bhadra P, Whalen E, Resnick EM. Effect of pregabalin on sleep in patients with fibromyalgia and sleep maintenance disturbance: a randomized, placebo-controlled, 2-way crossover polysomnography study. Arthritis Care Res (Hoboken). 2012;64(4):597-606.

25. Turk DC, Okifuji A, Sinclair JD, Starz TW. Differential responses by psychosocial subgroups of fibromyalgia syndrome patients to an interdisciplinary treatment. Arthritis Care Res. 1998;11(5):397-404.

26. Boomershine CS, Crofford LJ. A symptom-based approach to pharmacologic management of fibromyalgia. Nat Rev Rheumatol. 2009;5(4):191-199.

27. Fitzcharles MA, Ste-Marie PA, Goldenberg DL, et al. National fibromyalgia guideline advisory panel. Canadian guidelines for the diagnosis and management of fibromyalgia syndrome: executive summary. Pain Res Manag. 2013;18(3):119-126.

28. Piechan JL, Donevan SD, Taylor CP, Dickerson MR, \& Li Z. Pregabalin, a novel anticonvulsant, analgesic and anxiolytic drug, exhibits classspecific alpha2-delta-1 and alpha2-delta-2 calcium channel subunit binding. Soc Neurosci. Abstr 30:115.11.

29. Errante LD, Petroff OA. Acute effects of gabapentin and pregabalin on rat forebrain cellular GABA, glutamate, and glutamine concentrations. Seizure. 2003;12(5):300-306.

30. Micheva KD, Buchanan J, Holz RW, Smith SJ. Retrograde regulation of synaptic vesicle endocytosis and recycling. Nat Neurosci. 2003;6(9):925-932.

31. Cunningham MO, Woodhall GL, Thompson SE, Dooley DJ, Jones RSG. Dual effects of gabapentin and pregabalin on glutamate release at rat entorhinal synapses in vitro. Eur J Neurosci. 2004;20(6):1566-1576.

32. Bockbrader HN, Radulovic LL, Posvar EL, et al. Clinical pharmacokinetics of pregabalin in healthy volunteers. $J$ Clin Pharmacol. 2010;50(8):941-950.
33. Chew ML, Alvey CW, Plotka A, et al. Pregabalin controlled-release pharmacokinetics in healthy volunteers: analysis of four multipledose randomized clinical pharmacology studies. Clin Drug Investig. 2014;34(9):627-637.

34. Smart A. A multi-dimensional model of clinical utility. Int J Qual Health Care. 2006;18(5):377-382.

35. Crofford LJ, Rowbotham MC, Mease PJ, et al. Pregabalin for the treatment of fibromyalgia syndrome: results of a randomized, double-blind, placebo-controlled trial. Arthritis Rheum. 2005;52(4):1264-1273.

36. Arnold LM, Russell IJ, Diri EW, et al. A 14-week, randomized, doubleblinded, placebo-controlled monotherapy trial of pregabalin in patients with fibromyalgia. J Pain. 2008;9(9):792-805.

37. Mease PJ, Russell IJ, Arnold LM, et al. A randomized, double-blind, placebo-controlled, phase III trial of pregabalin in the treatment of patients with fibromyalgia. J Rheumatol. 2008;35(3):502-514.

38. Pauer L, Winkelmann A, Arsenault P, et al. An international, randomized, double-blind, placebo-controlled, phase III trial of pregabalin monotherapy in treatment of patients with fibromyalgia. $J$ Rheumatol. 2011;38(12):2643-2652.

39. Ohta H, Oka H, Usui C, Ohkura M, Suzuki M, Nishioka K. A randomized, double-blind, multicenter, placebo-controlled phase III trial to evaluate the efficacy and safety of pregabalin in Japanese patients with fibromyalgia. Arthritis Res Ther. 2012;14(5):R217.

40. Crofford LJ, Mease PJ, Simpson SL, et al. Fibromyalgia relapse evaluation and efficacy for durability of meaningful relief (FREEDOM): a 6-month, double-blind, placebo-controlled trial with pregabalin. Pain. 2008;136(3):419-431.

41. Ohta H, Oka H, Usui C, Ohkura M, Suzuki M, Nishioka K. An openlabel long-term phase III extension trial to evaluate the safety and efficacy of pregabalin in Japanese patients with fibromyalgia. Mod Rheumatol. 2013;23(6):1108-1115.

42. Farrar JT, Young JP Jr, LaMoreaux L, Werth JL, Poole RM. Clinical importance of changes in chronic pain intensity measured on an 11-point numerical pain rating scale. Pain. 2001;94(2):149-158.

43. Straube S, Derry S, Moore RA, McQuay HJ. Pregabalin in fibromyalgia: meta-analysis of efficacy and safety from company clinical trial reports. Rheumatology (Oxford). 2010;49(4):706-715.

44. Moore RA, Straube S, Wiffen PJ, Derry S, McQuay HJ. Pregabalin for acute and chronic pain in adults. Cochrane Database Syst Rev. 2009(3):CD007076.

45. Hindmarch I, Dawson J, Stanley N. A double-blind study in healthy volunteers to assess the effects on sleep of pregabalin compared with alprazolam and placebo. Sleep. 2005;28(2):187-193.

46. Zisapel N, Nir T. Determination of the minimal clinically significant difference on a patient visual analog sleep quality scale. J Sleep Res. 2003;12(4):291-298.

47. Russell IJ, Crofford LJ, Leon T, et al. The effects of pregabalin on sleep disturbance symptoms among individuals with fibromyalgia syndrome. Sleep Med. 2009;10(6):604-610.

48. Cappelleri JC, Bushmakin AG, McDermott AM, et al. Measurement properties of the medical outcomes study sleep scale in patients with fibromyalgia. Sleep Med. 2009;10(7):766-770.

49. Arnold LM, Emir B, Pauer L, Resnick M, Clair A. Time to improvement of pain and sleep quality in clinical trials of pregabalin for the treatment of fibromyalgia. Pain Med. 2015;16(1):176-185.

50. Pauer L, Atkinson G, Murphy TK, Petersel D, Zeiher B. Long-term maintenance of response across multiple fibromyalgia symptom domains in a randomized withdrawal study of pregabalin. Clin J Pain. 2012;28(7):609-614.

51. Straube S, Moore RA, Paine J, et al. Interference with work in fibromyalgia: effect of treatment with pregabalin and relation to pain response. BMC Musculoskelet Disord. 2011;12:125.

52. Arnold LM, Emir B, Murphy TK, et al. Safety profile and tolerability of up to 1 year of pregabalin treatment in 3 open-label extension studies in patients with fibromyalgia. Clin Ther. 2012;34(5): 1092-1102. 
53. Fong T, Lee AJ. Pregabalin-associated heart failure decompensation in a patient with a history of stage I heart failure. Ann Pharmacother. 2014;48(8):1077-1081.

54. Erdoğan G, Ceyhan D, Güleç S. Possible heart failure associated with pregabalin use: case report. Agri. 2011;23(2):80-83.

55. Page RL, Cantu M, Lindenfeld J, Hergott LJ, Lowes BD. Possible heart failure exacerbation associated with pregabalin: case discussion and literature review. J Cardiovasc Med (Hagerstown). 2008;9(9): 922-925.

56. Ho JM, Tricco AC, Perrier L, Chen M, Juurlink DN, Straus SE. Risk of heart failure and edema associated with the use of pregabalin: a systematic review. Syst Rev. 2013;2:25.

57. Pfizer Inc. Pregabalin (Lyrica) package insert. New York, NY: Pfizer; 2007.

58. Lloyd A, Boomershine CS, Choy EH, Chandran A, Zlateva G. The cost-effectiveness of pregabalin in the treatment of fibromyalgia: US perspective. J Med Econ. 2012;15(3):481-492.
59. Choy E, Richards S, Bowrin K, et al. Cost effectiveness of pregabalin in the treatment of fibromyalgia from a UK perspective. Curr Med Res Opin. 2010;26(4):965-975.

60. Neumann PJ, Cohen JT, Weinstein MC. Updating cost-effectiveness the curious resilience of the US\$50,000-per-QALY threshold. $N$ Engl J Med. 2014;371(9):796-797.

61. Keshavarz K, Hashemi-Meshkini A, Gharibnaseri Z, Nikfar S, Kebriaeezadeh A, Abdollahi M. A systematic cost-effectiveness analysis of pregabalin in the management of fibromyalgia: an Iranian experience. Arch Med Sci. 2013;9(6):961-967.

62. Arreola Ornelas H, Rosado Buzzo A, García L, Dorantes Aguilar J, Contreras Hernández I, Mould Quevedo JF. Cost-effectiveness analysis of pharmacologic treatment of fibromyalgia in Mexico. Reumatol Clin. 2012;8(3):120-127.

63. Parker L, Huelin R, Khankhel Z, Wasiak R, Mould J. A systematic review of pharmacoeconomic studies for pregabalin. Pain Pract. 2015;15(1):82-94.
Drug, Healthcare and Patient Safety

\section{Publish your work in this journal}

Drug, Healthcare and Patient Safety is an international, peer-reviewed open-access journal exploring patient safety issues in the healthcare continuum from diagnostic and screening interventions through to treatment, drug therapy and surgery. The journal is characterized by the rapid reporting of reviews, original research, clinical, epidemiological and

\section{Dovepress}

post-marketing surveillance studies, risk management, health literacy and educational programs across all areas of healthcare delivery. The manuscript management system is completely online and includes a very quick and fair peer-review system. Visit http://www.dovepress.com/ testimonials.php to read real quotes from published authors.

Submit your manuscript here: http://www.dovepress.com/drug-healthcare-and-patient-safety-journal 